\title{
Co-infecção humana pelo plasmódio e o vírus da hepatite B: aspectos clínicos, sorológicos e imunológicos
}

\author{
Coinfection between hepatitis B virus and malaria: clinical, serologic and \\ immunologic aspects
}

\author{
Wornei Silva Miranda Braga ${ }^{1}$, Rita Auxiliadora Botelho de Souza ${ }^{1}$, Eva Batista da Silva ${ }^{1}$, \\ José Carlos Ferraz da Fonseca ${ }^{1}$ e Carlos Eduardo Tosta ${ }^{2}$
}

\begin{abstract}
RESUMO
A Amazônia é conhecida pela elevada prevalência de infecção pelo vírus da hepatite B, contribui também com mais de 90\% dos casos de malária do país. É proposto que a ocorrência de co-infecções seja importante e que na associação ocorram alterações na história natural dessas enfermidades. O estudo avalia 545 pacientes com malária, em Coari, AM: 333 (61,1\%) pelo Plasmodium vivax, 193 (35,4\%) pelo Plasmodium falciparum e 19 (3,5\%) com infecção mista. A prevalência do AgHBs foi 4,2\% e a do anti-HBc total 49,7\%. Os pacientes sororreativos para o VHB, não apresentaram diferenças clínicas dos outros pacientes com malária, nem associação a sinais clássicos de comprometimento hepático. Apesar de não ter sido detectada associação estatisticamente significativa, os indivíduos $\mathrm{AgHBs}$ reativos apresentaram baixas parasitemias e índices de reatividade de anticorpos mais elevados, sugerindo a possibilidade da resposta imune em um indivíduo co-infectado ser diferenciada e favorecer variações em relação à parasitemia e produção de anticorpos.
\end{abstract}

Palavras-chaves: Hepatite B. Malária. Co-infecção. Amazônia.

\section{ABSTRACT}

The Amazon region is known for a high prevalence of hepatitis B infection, and accounts for more than 90\% of malaria cases in Brazil. It has been suggested that the occurrence of coinfections may be important, and may influence the natural history of both diseases. This study evaluated 545 patients with acute malaria, in Coari, Western Brazilian Amazon. 333 (61.1\%) presented Plasmodium vivax malaria, 193 (35.4\%) Plasmodium falciparum and 19 (3.5\%) mixed infections. The HBsAg prevalence was 4.2\% and total anti-HBC 49.7\%. Patients with HBV serological markers presented no clinical differences than those with malaria only, nor showed any association with classic signs of hepatic disorder. Although showing no statistical significance, HBSAg reactive subjects presented lower parasitic load and higher antibody titers, suggesting the possibility that the immune response in a coinfected individual is differentiated and leads to a variation in the parasite load and antibody production.

Key-words: Hepatitis B. Malaria. Coinfections. Amazon.

Estima-se que aproximadamente $1 / 3$ da população mundial já teve contacto com o vírus da hepatite B (VHB) e que cerca de 500 milhões de indivíduos sejam portadores do antígeno de superfície viral $(\mathrm{AgHBs})^{12}{ }^{23}$. A região Amazônica é caracterizada como uma das regiões de maior ocorrência de infecção pelo VHB no planeta ${ }^{24}$.

Cerca de $40 \%$ da população mundial vivem em área de risco de transmissão de malária, estima-se a ocorrência de cerca de 500 milhões de casos a cada ano e mais de dois milhões de mortes $^{22}$. No Brasil, a região amazônica contribui com a grande maioria do número de casos, mais de $90 \%$, em 2000, cerca de 608 mil casos (Informes Técnicos Fundação Nacional de Saúde, 1999).

A associação do VHB com outros vírus hepatotrópicos resulta no desenvolvimento de formas graves de hepatite $\mathrm{e}$ na evolução precoce para doença crônica ${ }^{24}$.

Têm sido descritas associações da infecção por malária com salmolella ${ }^{11}$, reposta inadequada à vacinas ${ }^{13}$, reação

1. Fundação de Medicina Tropical do Amazonas, Manaus, Manaus, AM. 2. Laboratório de Malária do Núcleo de Medicina Tropical da Faculdade de Medicina da Universidade de Brasília, DF.

Endereço para correspondência: Dr. Wornei Braga. Fundação de Medicina Tropical do Amazonas. Av. Pedro Teixeira 25, D. Pedro I, 69040-000 Manaus, AM. Fax: 5592 238-3762

e-mailwbraga@fmt.am.gov.brouwornei@hotmail.com

Recebido para publicação em 20/4/2004

Aceito em 23/9/2005 
cruzada do Plasmodium falciparum com anticorpos contra 0 vírus humano linfotrópico de células $\mathrm{T}^{9}$, e com outras enfermidades como sarampo e influenza ${ }^{18}$. Na África, em regiões de elevada ocorrência de malária por $P$. falciparum, observa-se elevada prevalência de linfoma de Burkitt, sugerindo que esta facilitaria a expressão da oncogenicidade do vírus Epstein-Barr ${ }^{21}$.

Em estudos experimentais foi demonstrada a supressão do gene do VHB e sua replicação, em camundongos transgênicos co-infectados pelo VHB e Plasmodium yoelii ${ }^{17}$.

As regiões do planeta de maior ocorrência de malária e, de hepatite B são justapostas, no entanto, tem sido pouco estudada a associação do VHB com a malária. Apesar da controvérsia, é proposto que quando associados o VHB e a malária, alterações imunológicas influenciariam na história natural dessas enfermidades ${ }^{51014}$, objeto da presente investigação.

\section{MATERIAL E MÉTODOS}

Foi conduzido estudo descritivo de uma série de casos de malária aguda, com objetivo de investigar os aspectos clínicos, bioquímicos e imunológicos, mais importantes, na associação entre a malária e o VHB. Os pacientes foram selecionados da demanda espontânea do serviço de malária da Fundação de Medicina Tropical do Amazonas (FMTAM), na Cidade de Coari, região do médio Solimões, no período de maio de 2001 a março de 2002 (Figura 1).

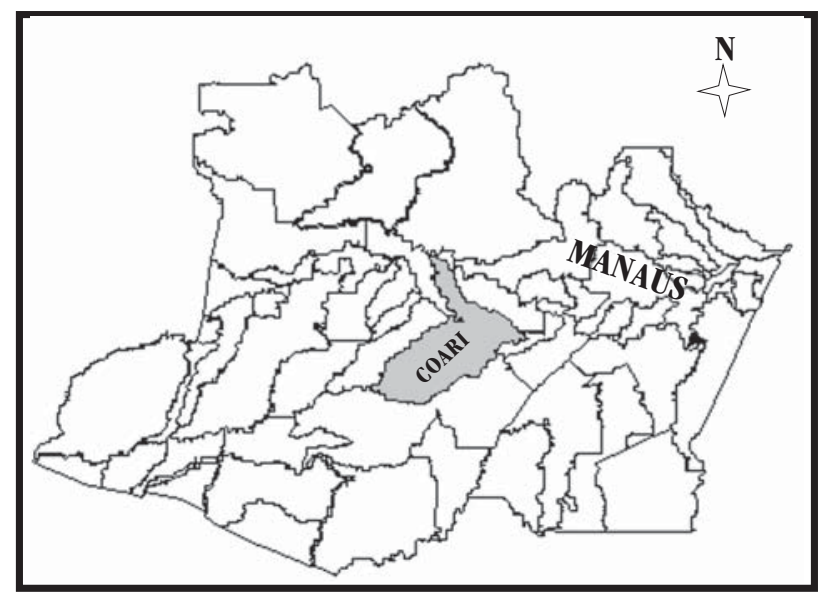

Figura 1 - Mapa do Estado do Amazonas, com destaque para a região de estudo.

Coari foi escolhida devido à intensidade de transmissão malárica, e pela elevada endemicidade de infecção pelo $\mathrm{VHB}^{4}$. Em 2000, o município foi responsável por mais de $40 \%$ dos casos de malária do Estado do Amazonas, com índice parasitário anual (IPA) de 113,3 casos por 1.000 habitantes e percentual de infecção por Plasmodium falciparum de 18,8\% (Fundação de Medicina Tropical do Amazonas, Boletim Trimestral outubro, 1999).

Os casos de co-infecção entre o VHB e a malária consistiram em dois grupos: 1) pacientes com malária, cursando com sorologia reativa para o AgHBs; e 2) pacientes com malária com sorologia positiva para 0 anti-HBc total.
Foram excluídas gestantes, menores de 14 anos de idade, alcoólatras, em uso prévio de medicação hepatotóxica, citotóxica ou imunossupressora, e portadores de enfermidades crônicas, para amenizar efeitos de fatores que pudessem já estar associados a dano hepático, ou risco potencial de malária atípica. 0 protocolo foi autorizado pelo Comitê de Ética em Pesquisa da FMTAM. As informações foram registradas em um questionário padrão e coletada uma amostra de $10 \mathrm{ml}$ de sangue, na primeira consulta, pelo sistema vacuotainer.

A sorologia do VHB foi realizada pelo método imunoenzimático (ELISA), utilizando-se kits comerciais (Organon Teknica®), obedecendo normas do fabricante. Todas as amostras foram testadas para o AgHBs e anti-HBc total.

As amostras foram testadas para a presença de anticorpos IgM e IgG, pelo método imunoenzimático (ELISA), com antígeno de formas sanguíneas do $P$. falciparum, obtidos por cultivo in vitro e do $P$. vivax a partir de sangue de pacientes infectados, atendidos na FMTAM. As amostras de soro testadas foram classificadas em reativas e não reativas e avaliados os níveis de anticorpos medidos pelo índice de reatividade (IR = densidade óptica da amostra/ densidade óptica do ponto de corte da reação).

A análise dos dados foi iniciada com descrição estatística simples, incluindo distribuição dos tipos de plasmódio, prevalência de marcadores do VHB, idade, sexo e outras variáveis. Transformação logarítmica e cálculo de médias geométricas foram empregados na análise da parasitemia e índice de reatividade médio de anticorpos.

\section{RESULTADOS}

0 estudo incluiu 545 pacientes com quadro de malária aguda, $410(75,2 \%)$ do sexo masculino, e com uma mediana de idade de 27 anos (14-69). Destes, 333 (61,1\%) apresentavam malária pelo $P$. vivax, $193(35,4 \%)$ pelo $P$. falciparum e 19 (3,5\%) pelo $P$. vivax associado ao P. falciparum. Em relação a infecções prévias, 117 (21,5\%) eram primoinfectados e $428(78,5 \%)$ tinham história de um ou mais episódios de malária anterior.

A prevalência do AgHBs no grupo de estudo foi 4,2\% (23/ $545)$ e a prevalência do anti-HBc total foi $49,7 \%$ (271/545) (Tabela 1).

Aspectos clínicos. Os sinais e sintomas mais freqüentemente encontrados foram: febre $(94,9 \%)$, cefaléia $(92,3 \%)$, artralgia $(83,9 \%)$ e calafrios $(82 \%)$. Nenhum dos

Tabela 1 - Prevalência de marcadores sorológicos do VHB por tipo de malária em 545 pacientes examinados em Coari, Amazonas, 2001-2002.

\begin{tabular}{|c|c|c|c|c|c|c|}
\hline \multirow[b]{2}{*}{ Tipo de malária } & \multicolumn{2}{|c|}{$\mathrm{AgHBs}$} & \multicolumn{2}{|c|}{ Anti-HBc total } & \multicolumn{2}{|c|}{ Total } \\
\hline & $\mathrm{n}^{\underline{0}}$ & $\% a$ & $\mathrm{n}^{\mathrm{0}}$ & $\% a$ & $\mathrm{n}^{0}$ & $\% \mathrm{~b}$ \\
\hline Plasmodium vivax & 12 & 3,6 & 155 & 46,5 & 333 & 61,1 \\
\hline Plasmodium falciparum & 9 & 4,7 & 101 & 52,3 & 193 & 35,4 \\
\hline Associada & 2 & 10,5 & 15 & 78,9 & 19 & 3,5 \\
\hline Total & 23 & 4,2 & 271 & 49,7 & 545 & 100,0 \\
\hline
\end{tabular}

a. Percentual do total de cada grupo (tipo de infecção) b. Percentual do total de indivíduos estudados. 
pacientes atendidos apresentou quadro clínico que os definissem como casos de malária grave. Não encontramos diferença estatisticamente significativa da presença de marcadores sorológicos de infecção pelo VHB e manifestações clínicas clássicas da malária. 0 achado de hepatomegalia e de esplenomegalia, no primeiro exame físico, não mostrou associação com a presença de marcador do VHB (Tabela 2).

Níveis séricos de alaninoaminotransferase. 0 nível médio de alaninoaminotransferase (ALT), medido em 440 dos pacientes incluídos foi 34U/L (10-223U/L). Estabelecendo um valor de referência de até 65U/L como padrão de normalidade, encontramos uma prevalência de 9,5\% (42/440) de pacientes com níveis elevados de ALT.

Nos pacientes com história de um ou mais episódios prévios de malária a taxa de prevalência de ALT elevada foi
$7,3 \%$ (25/344) e nos primoinfectados 17,7\% (17/96) com um risco relativo $(\mathrm{RR})$ igual a $\mathrm{RR}=2,44(\mathrm{p}=0,003)$ (Tabela 3). A presença do AgHBs, mesmo ajustada por número de episódios, mostrou-se fator de risco de ALT elevada, $\mathrm{RR}=3,02(\mathrm{p}=0,01)$ (Tabela 3).

Níveis de parasitemia. Foi avaliada a parasitemia de 428 pacientes, variando de 1.107 parasitas/ $\mu 1$ entre os pacientes com malária pelo $P$. vivax a 1.596 parasitas/ $\mu 1$, entre os pacientes com malária pelo $P$. Falciparum (Tabela 4) $(\mathrm{p}=0,007$, ANOVA). Os pacientes com história de um ou mais episódios anteriores, em média, apresentaram 1.449 parasitas/ $\mu \mathrm{l}$ e os pacientes primoinfectados 777 parasitas/ $\mu 1$ $(\mathrm{p}<0,05$, ANOVA) (Tabela 4).

Os pacientes portadores do AgHBs apresentaram, em média, menor densidade parasitária, embora esta variação não tenha se mostrado estatisticamente significativa (Tabela 4).

Tabela 2 - Parâmetros clínicos de malária em relação à presença do AgHBs e ou anti-HBe total isolado em 545 pacientes com malária examinados em Coari, Amazonas, 2001-2002.

\begin{tabular}{|c|c|c|c|c|c|c|c|}
\hline \multirow[b]{2}{*}{ Parâmetro } & \multicolumn{3}{|c|}{$\begin{array}{l}\text { Marcador do } \\
\text { VHB }^{\mathrm{a}} \text { presente }\end{array}$} & \multirow{2}{*}{$\begin{array}{l}\text { Marcador do } \\
\text { VHB ausente } \\
\text { média ou \% }\end{array}$} & \multirow{2}{*}{$\begin{array}{l}\text { RP ou diferença } \\
\text { entre médias }\end{array}$} & \multirow[b]{2}{*}{ IC-95\% } & \multirow[b]{2}{*}{$\mathrm{P}$} \\
\hline & $\mathrm{n}^{0}$ & média ou \% & $\mathrm{n}^{0}$ & & & & \\
\hline Dias de doença & 271 & 4,9 dias & 274 & 4,5 dias & 0,4 dias & $0-0,8$ & 0,14 \\
\hline Clínica clássica ${ }^{\mathrm{b}}$ & $218 / 271$ & $80,4 \%$ & $211 / 274$ & $77,0 \%$ & 1,04 & $0,86-1,38$ & 0,38 \\
\hline Hepatomegalia & $78 / 265$ & $29,4 \%$ & $69 / 268$ & $25,7 \%$ & 1,14 & $0,91-1,32$ & 0,39 \\
\hline Esplenomegalia & $88 / 265$ & $33,2 \%$ & $86 / 268$ & $32,1 \%$ & 1,03 & $0,86-1,38$ & 0,85 \\
\hline Taxa de negativação & $181 / 271$ & $66,8 \%$ & $168 / 274$ & $61,3 \%$ & 1,09 & $0,94-1,36$ & 0,21 \\
\hline
\end{tabular}

$\mathrm{N}$, tamanho da amostra em cada grupo; RP, razão de prevalência; IC, intervalo de confiança; p, significância estatística

a. AgHBs ou anti-HBc total isolado;

b. Febre, cefaléia, sudorese e calafrios

Tabela 3 - Fatores associados à elevação dos níveis séricos de ALT era 440 pacientes com malária examinados em Coari, Amazonas, 2001-2002.

\begin{tabular}{lrrrrrrrrr}
\hline & \multicolumn{2}{c}{ Fator presente } & & \multicolumn{2}{c}{ Fator ausente } & & & \\
\cline { 2 - 3 } Fator & $\mathrm{n}^{0}$ & $\%$ & & $\mathrm{n}^{0}$ & $\%$ & RP & IC-95\% & $\mathrm{p}$ \\
\hline Episódios $^{\mathrm{a}}\left(\mathrm{n}^{\mathrm{0}}\right)$ & $17 / 96$ & 17,7 & & $25 / 344$ & 7,3 & 2,44 & $1,37-4,32$ & 0,003 \\
AgHBs $^{\mathrm{b}}$ & $6 / 23$ & 26,1 & & $36 / 417$ & 8,6 & 3,02 & $1,41-6,49$ & 0,01 \\
Anti-HBc total $^{\mathrm{b}}$ & $25 / 224$ & 11,2 & & $17 / 216$ & 7,9 & 1,42 & $0,80-2,52$ & 0,30
\end{tabular}

$\mathrm{N}$, tamanho da amostra em cada grupo; RP, razão de prevalência; IC, intervalo de confiança; p, significância estatística.

a. Razão entre a prevalência de ALT elevada nos primoinfectados em relação aos reinfectados;

b. Ajustado por $n^{\circ}$ de episódios prévios.

Tabela 4 - Diferença da parasitemia média era relação à espécie de plasmódio, número de malárias pregressas e marcadores do VHB em 428 pacientes com malária examinados em Coari, Amazonas, 2001-2002.

\begin{tabular}{lcccccc}
\hline & & & \multicolumn{3}{c}{ Diferença } \\
Fator & № & Média & № & Média & entre médias & $\mathrm{P}$ \\
\hline Espécie de plasmódio $^{a}$ & 165 & 1.596 parasitas/Pl & 263 & 1.107 parasitas/Pl & 489 parasitas/Pl & 0,007 \\
Episódios $^{\text {b }}\left(\mathrm{n}^{\mathrm{o}}\right)$ & 28 & 777 parasitas/Pl & 340 & 1.449 parasitas/Pl & -672 parasitas/Pl & $<0,03$ \\
AgHBs $^{c}$ & 22 & 1.171 parasitas/Pl & 406 & 1.280 parasitas/Pl & -109 parasitas/Pl & 0,46 \\
Anti-HBc total $^{c}$ & 216 & 1.355 parasitas/Pl & 212 & 1.198 parasitas/Pl & 157 parasitas/Pl & 0,52 \\
\hline
\end{tabular}

$\mathrm{N}$, tamanho da amostra em cada grupo; Média, média geométrica; IC, intervalo de confiança; p, significância estatística; AgHBs, antígeno de superfície do vírus da hepatite B; anti-HBe total, anticorpo contra o antígeno do core do VHB.

a. Diferença entre a parasitemia média dos infectados pelo $P$. falciparum e os infectados pelo $P$. vivax;

b. Diferença entre a parasitemia média dos primoinfectados e reinfectados;

c. Diferença entre a parasitemia média dos reativos e não reativos 
Imunoglobulinas antiPlasmodium. Nos indivíduos apresentando o $\mathrm{AgHBs}$, os IR médios de $\mathrm{IgG}$ anti-P. falciparum variaram de 1,420 nos pacientes reativos e 1,215 nos não reativos ( $\mathrm{p}=0,35$, ANOVA) (Tabela 5 e Figura 2). Os IR médios de IgM antiP. falciparum variaram de 1,331 nos portadores do AgHBs e de 1,242 nos não reativos ( $\mathrm{p}=0,37, \mathrm{ANOVA}$ ) (Tabela 5). Os IR médios de IgG anti- $P$. vivax variaram de 1,667 nos pacientes reativos para o AgHBs e 1,618 nos não reativos ( $\mathrm{p}=0,85$, ANOVA) (Tabela 5). Os IR médios de IgM anti-P. vivax variaram de 1,338 nos pacientes portadores do AgHBs e de 1,460 nos não reativos ( $\mathrm{p}=0,41$, ANOVA) (Tabela 5).

\begin{tabular}{|c|c|c|c|c|c|c|}
\hline \multirow[t]{2}{*}{ Fator/Imunoglobulina } & \multicolumn{2}{|c|}{ Presente } & \multicolumn{2}{|c|}{ Ausente } & \multirow{2}{*}{$\begin{array}{c}\text { Diferença entre } \\
\text { médias }^{\mathrm{a}}\end{array}$} & \multirow[b]{2}{*}{$\mathrm{P}$} \\
\hline & $\mathrm{n}^{\mathrm{o}}$ & média & $\overline{\mathrm{n}^{0}}$ & média & & \\
\hline \multicolumn{7}{|l|}{$\mathrm{AgHBs}$} \\
\hline IgG anti-P. falciparum & 9 & 1.420 & 183 & 1.215 & 0,205 & 0,35 \\
\hline IgM anti-P. falciparum & 9 & 1.331 & 179 & 1.242 & 0,089 & 0,37 \\
\hline IgG anti-P. vivax & 12 & 1.667 & 321 & 1.618 & 0,049 & 0,85 \\
\hline IgM anti-P. vivax & 11 & 1.338 & 306 & 1.460 & $-0,122$ & 0,41 \\
\hline \multicolumn{7}{|l|}{ Anti-HBc total } \\
\hline $\operatorname{IgG}$ anti-P. falciparum 1 & 101 & 1.222 & 91 & 1.227 & $-0,005$ & 0,87 \\
\hline IgM anti-P. falciparum & 99 & 1.222 & 89 & 1.272 & $-0,05$ & 0,52 \\
\hline IgG anti-P. vivax & 155 & 1.619 & 178 & 1.621 & $-0,002$ & 0,87 \\
\hline IgM anti-P. vivax & 149 & 1.430 & 168 & 1.479 & $-0,049$ & 0,91 \\
\hline
\end{tabular}

N, tamanho da amostra em cada grupo; Média, média geométrica; $p$, significância estatística; $\mathrm{AgHBs}$, antígeno de superfície do vírus da hepatite $\mathrm{B}$; anti-HBc total, anticorpo contra 0 antígeno do core do VHB. ${ }^{\text {a }}$ Diferença entre os IR médios dos primoinfectados e reinfectados.

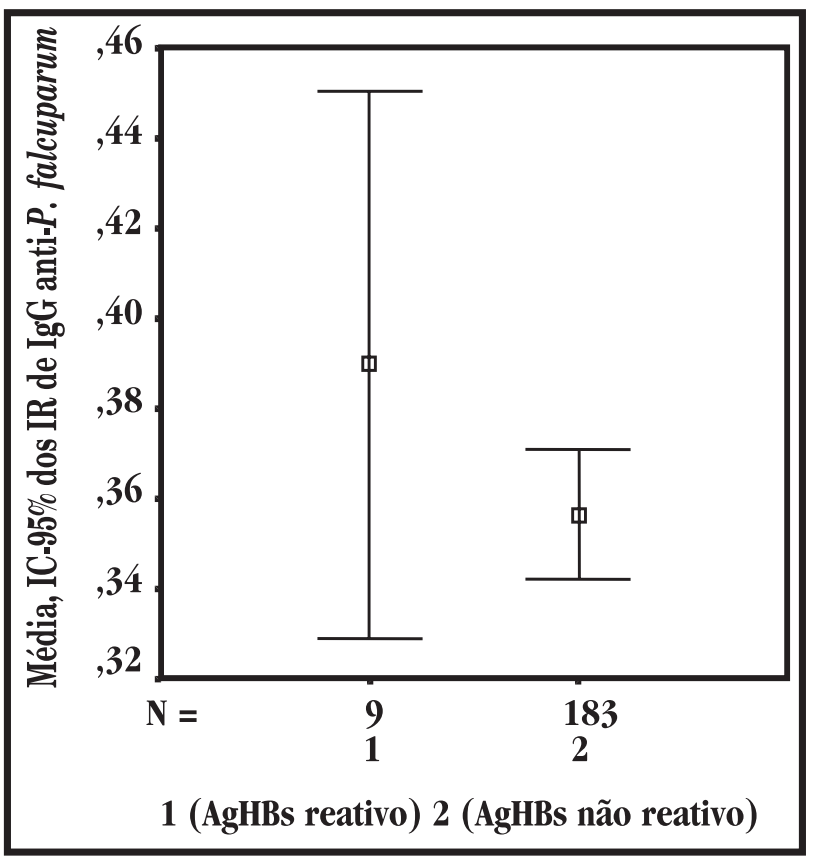

Figura 2 - Intervalo de confiança de 95\% da média dos Índices de Reatividade de IgG anti-P. falciparum (escala logarítmica) na presença do AgHBs em pacientes com malária atendidos em Coari, Amazonas, 2001-2002.

\section{DISCUSS̃̃o}

Encontramos predominância de casos de malária por P. vivax, característica atual da malária na Amazônia Brasileira ${ }^{16}$. Apesar de ser considerada área de transmissão instável, 78,5\% dos pacientes referiam quadros anteriores de malária, 54,9\% deles no mesmo ano da inclusão no estudo, caracterizando período de intensa transmissão. As taxas de prevalência de marcadores do VHB nos pacientes avaliados se assemelham às encontradas em outras regiões endêmicas do estado $0^{4}$.

Não houve diferença estatisticamente significativa entre a presença de marcadores de infecção presente ou passada pelo VHB e quadro clínico de malária, inclusive com sinais clássicos de comprometimento hepático, hepatomegalia, icterícia ou colúria, indicando que a presença do VHB, em atividade, ou de anticorpos anti-VHB, provavelmente não influenciaram o curso do quadro clínico dos pacientes avaliados. Outros estudos associam a presença do VHB com quadros de malária grave ${ }^{1620}$. Acreditamos que a malária grave e complicada caracterizaria situação distinta, com fatores determinantes diversos, em que a associação com o VHB deva ser avaliada sob outro angulo.

Os níveis séricos de ALT elevados se mostraram associados à primoinfecção, provavelmente pela evolução da infecção pelo plasmódio, nestes indivíduos. A ação direta do plasmódio sobre o hepatócito, é questionada, já que os maiores danos descritos, associados à presença do plasmódio, ocorrem nos sinusóides hepáticos com pouca lesão de hepatócitos ${ }^{15}$, onde ocorre intensa atividade inflamatória, que leva à hipóxia tecidual e aumento da permeabilidade da membrana do hepatócito com liberação de ALTT $^{719}$.

Na presença de AgHBs, o risco de ALT elevada foi cerca de três vezes maior, mesmo depois de ajustado por número de episódios. No entanto, não foi possível estabelecer relação com a infecção pelo plasmódio, sem o perfil bioquímico anterior ao quadro. Como na região, a presença de ALT elevada é associada ao AgHBs em população assintomática ${ }^{4}$, essas alterações podem resultar da infecção crônica pelo VHB.

$O s$ pacientes infectados pelo $P$. falciparum apresentavam, em média, maior densidade parasitária que os infectados pelo $P$. vivax, o que é descrito na literatura, pois o $P$. falciparum não seleciona hemácias jovens como o $P$. vivax e possui ciclo eritrocitário mais curto ${ }^{8}$.

Em média os portadores de AgHBs apresentaram densidades parasitárias menores e índices de reatividade de anticorpos mais elevados, embora sem diferença estatística significativa. Acreditamos que o tamanho da amostra pode ter influenciado nos resultados negativos.

0 conceito de hepatite na malária é empregado de maneira heterogênea. É definida com os seguintes critérios: 1) clínicos, identificando-a em pacientes com malária cursando com hepatomegalia e icterícia; 2) bioquímicos, quando os pacientes apresentam elevação dos níveis de aminotransferases séricas ou 3) histopatológicos com a presença de necrose centrolobular e congestão de sinusóides hepáticos ${ }^{310}$. Em nosso estudo o perfíl do paciente com hepatite identificado foi o daquele indivíduo com malária, independente de sexo, espécie de plasmódio, idade ou níveis de parasitemia, com história de malária pela primeira vez ou portador crônico do VHB.

A importância da associação entre o VHB e o plasmódio, não deve estar relacionada com o número de casos de co- 
infecções, em área endêmica dessas enfermidades, nem com sua expressão clínica durante um episódio de malária leve ou moderada. Outros fatores merecem ser investigados como a possibilidade da resposta imune em um indivíduo coinfectado ser diferenciada e favorecer diferenças em relação à parasitemia e produção de anticorpos.

\section{REFERÊNCIAS BIBLIOGRÁFICAS}

1. Barcus MJ, Hien TT, White NJ, Laras K, Farrar J, Schwartz IK, Corwin A, Baird JK. Short Report: Hepatitis B infection and severe Plasmodium falciparum malaria in Vietnamese adults. The American Journal of Tropical Medicine and Hygiene 66:140-142, 2002.

2. Bensabath G, Hadler SC, Soares MCP, Fields H, Dias LB, Popper H, Maynard JE. Hepatitis Delta virus infection and Lábrea hepatitis. Prevalence and role in fulminant hepatitis in the Amazon basin. Journal American Medical Association 258:479-483, 1987.

3. Boulos M. Comprometimento hepático na malária. Correlação clínica, bioquímica, patológica. Tese de doutorado. Universidade de São Paulo, São Paulo, 1983.

4. Braga WSM, Brasil LM, Souza RAB, Melo MS, Rosas MDG, Castilho MC, Fonseca JCF. Prevalência da infecção pelos vírus da hepatite B (VHB) e hepatite Delta (VHD) em Lábrea, rio Purus, Estado do Amazonas. Epidemiologia e Serviços de Saúde 13:35-46, 2004.

5. Brown AE, Mogkolsirichaikul D, Innis B, Snitbhan R, Webster HK. Falciparum malaria modulates viremia in chronic hepatitis B virus infection. Journal of Infectious Diseases 166:1465-1466, 1992

6. Deloron P, Ringwald P, Luty AJ, Renaut A, Minh TN, Mbessy JR, Millet P. Relationship between malaria prevalence and malaria-related morbidity in school children from two villages in Central Africa. The American Journal of Tropical Medicine and Hygiene 61:99-102, 1999.

7. Frevert U. Malaria sporozoite-hepatocyte interactions. Experimental Parasitology 79:206-210, 1994.

8. Gilles HM, Warrell DA. Bruce-Chwatt's Essential Malariology. Edward Arnold, London, 1993.

9. Hayes CG, Burans JP, Obsert RB. Antibodies to human T lymphotropic virus type 1 in a population from the Philippines: Evidence for cross-reactivity with Plasmodium falciparum. Journal of Infectious Diseases 163: 257-262, 1991.

10. Hollingdale MR. Malaria and the liver. Hepatology 5:327-335, 1985.

11. Mabey DC, Brown A, Greenwood BM. Plasmodium falciparum malaria and Salmonella infections in Gambian children. Journal of Infectious Diseases 155:1319-1321, 1987.

12. Maynard JE. Hepatitis B global importance and need for control. Vaccine 8(supl 1): S18-S20, 1990.

13. McGregor IA, Barr M. Antibody response to tetanus toxoid inoculation in malarious and non-malarious Gambian children. Transactions of the Royal Society of Tropical Medicine and Hygiene 56:364-367, 1962.

14. Misrha SK, Das BS, Patnaik JK, Satpathy SK, Mohanty D, Bose TK. Hepatic changes in $P$. falciparum malaria (English abstract). Indian Journal of Malariology 29:167-171, 1992.

15. Mota MM, Pradel G, Vanderberg JP, Hafalla JC, Frevert U, Nussenzweig RS, Nussenzweig V. Migration of Plasmodium sporozoites through cells before infection. Science 291:141-144, 2001.

16. Motta EF. Fatores determinantes da situação da malária na Amazônia. Revista da Sociedade Brasileira de Medicina Tropical 25(supl II):27-32, 1992

17. Pasquetto V, Guidotti LG, Kakimi K, Tsuji M, Chisari FV. Host-Virus Interactions during Malaria Infection in Hepatitis B virus Transgenic Mice. Journal of Experimental Medicine 192: 529-535, 2000

18. Rooth IB, Bjorkman A. Supression of Plasmodium falciparum infections during concomitant measles or influenza but not during pertusis. The American Journal of Tropical Medicine and Hygiene 47:675-681, 1992.

19. Sinnis P. The malaria sporozoite's journey into the liver. Infectious Agents and Disease 5:182-189, 1996.

20. Thursz MR, Kwiatkowski D, Torok ME, Allsopp CE, Greenwood BM, Whittle HC, Thomas HC, Hill AV. Association of hepatitis B surface antigen carriage with severe malaria in Gambian children. Nature Medicine 1:374-375, 1995.

21. Whittle HC, Brown J, Greenwood BM, Seidelin P, Tighe H, Wedderburn L. T cell control of Epstein-Barr virus infected cell is lost during Plasmodium falciparum malaria. Nature 312:449-450, 1984.

22. World Health Organization, Weekly Epidemiological Record, Malaria, 1982-1997. No. 32, 74:265-272, 1999.

23. World Health Organization. Hepatitis B Fact Sheet WHO/204. Disponível no site: http://www.who.int/inf-fs/en/fact204.html, 2001. 